\title{
Assessment of normal values of Area Strain by using Three-dimensional speckle-tracking Echocardiography
}

\author{
Ali Mohammed Al Amin, Mohammed Hisham Hassan, Yehia El Maadawy Mohammed, \\ Ashraf Al-Amir Abd Elfattah, Mostafa Tarek Mostafa*
}

\author{
Department of Cardiology, Faculty of Medicine, Al-Azhar University, Cairo, Egypt \\ *Corresponding author: Mostafa Tarek Mostafa, Email address: mustafa.tarek@live.com
}

\begin{abstract}
Background: area strain (AS) has become a parameter of increasing concern. AS is a parameter that combines both the longitudinal and the circumferential deformations and it is acquired using three dimensional speckle tracking echocardiography (3D STE). This parameter should be used to detect pathological changes as it is more sensitive than the conventional parameters in evaluating left ventricular (LV) systolic function. Aim: the present study aimed to estimate the maximum normal systolic reference value of Area Strain in a sample of healthy subjects. Subjects and

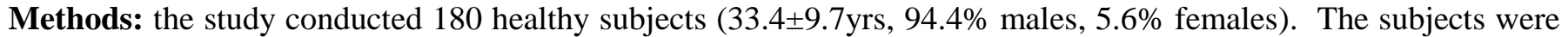
volunteers without heart-related diseases or other diseases. They were classified into three different age groups : Group I is between the ages of 20-30, Group II is between the ages of 30-40 and Group III is between the ages of 4050. Results: the study showed that the mean global area strain at group I, group II and group III were $-28.40 \pm 2.48 \%$ ,$-28.57 \pm 2.73 \%$ and $-28.53 \pm 2.81 \%$ respectively. The mean maximum systolic global area strain of all the studied subjects was $-28.50 \pm 2.66 \%$. The difference between the three groups was statistically insignificant. Conclusion: the study concluded that global area strain obtained from 3D STE may be used clinically as an additional diagnostic method since Area Strain can provide a more global and comprehensive assessment of LV systolic function than traditional parameters.
\end{abstract}

Keywords: Area Strain (AS), three dimensional (3D), speckle tracking echocardiography (STE)

\section{Introduction}

Strain echocardiography has become an established and important clinical evaluation technique for cardiac function. Myocardial strain has been shown toadd information on global and regional function and the timing of myocardial contraction beyond such conventional markers as LV ejection fraction (1). The area strain (AS) is a measurement of the relative percentage change in the area of a particular myocardial segment, representing the percentage change in the myocardial surface from its original dimensions (2). The three-dimensional speckle-tracking echocardiography (3D-STE) is a promising instrument to precisely quantify $\mathrm{LV}$ strain using complete $3 \mathrm{D}$ pyramidal data sets. Preliminary studies showed that 3D-STE may overcome the constraints of Doppler-based strain or 2D-based speckletracking strain images ${ }^{(3-5)}$. In addition, 3D strain analysis is angle independent and keeps away from strain estimation mistakes due to out-of-plane movement. At last, it gives new indices such as Area Strain and may enable more exact estimations of LV twist and shear strain (6).

Area tracking, known as Area Strain (AS), is a novel concept which is quantified by the percentage of deformation in the LV endocardial surface area using 3D-STE. Area Strain consolidates both longitudinal and circumferential deformations ${ }^{(7)}$. This gives an estimate of the subendocardial surface deformation that is inversely proportional to the radial deformation (systolic thickening and diastolic thinning) of the

wall of the ventricle, and is the first endocardium area to be affected by various cardiopathies. In other words, 3D tracking of myocardial displacement can be broken down into the three spatial axes: longitudinal, circumferential and radial, in the case of left ventricular myocardium. Comparing the displacement of two different points, information on myocardial deformation or strain can be obtained in each of these three different directions. You can see a surface by combining 2 of these directions. By consolidating longitudinal and circumferential deformation in a given myocardium segment, the deformation of subendocardial surface, which in this case is inversely proportional to the deformation of the myocardium in the third dimension, the radial one, can be acquired ${ }^{(8)}$. The study aim is to estimate the maximum normal systolic reference value of Area Strain in a sample of healthy subjects.

\section{Patients and Methods}

This prospective study was carried out on 180 consecutive subjects (mean age $=33.4 \pm 9.7 \mathrm{yrs}, 94.4 \%$ males, $5.6 \%$ females) in the Islamic Cardiac Center in Al-Azhar University during the period from January 2017 to September 2018. The subjects of this study were volunteers without heart-related diseases or other related diseases. They were classified into three different age groups: Group I is between the ages of 20-30, Group II is between the ages of 30-40 and Group III is between the ages of 
40-50. The study was approved by the Ethics Board of AlAzhar University.

\section{Inclusion criteria:}

- Age: 20-50 years old.

- Normal physical examination, electrocardiographic, and echocardiographic findings. Informed oral \& written consent to participate in the study.

- Normal laboratory results for complete blood picture, serum creatinine, blood urea nitrogen, serum uric acid, urine analysis, lipid profile and fasting blood sugar.

\section{Exclusion criteria:}

- Arrythmia, all types of Heart block, coronary artery disease, congenital \& valvular heart disease, cardiomyopathy \& Heart Failure.

- Systemic infection.

- Pregnancy.

- Hypertension \& Diabetes Mellitus.

- Suboptimal image quality unsuitable for strain measurements.

- Refusal to participate

All Subjects underwent the following:

- All subjects underwent a two-dimensional echocardiographic examination to exclude valvular and myocardial heart diseases, followed by a 3D-Echo examination using a Vivid E95 ultrasound system (GE Medical Systems). For the two-dimensional (2D) examination, we used a 1.4-4.6 MHz M5Sc probe, and for the $3 \mathrm{D}$ exam, a $1.5-4 \mathrm{MHz} 4 \mathrm{~V}$ probe (GE Medical Systems).

- $\quad 3 \mathrm{D}$ images with the 3D trans-thoracic probe were obtained from apical positions. Full volume acquisition involving the capture of four adjacent subvolumes over four consecutive cardiac cycles was performed during subject on breath hold to minimize the stitch artifact between the subvolumes. Depending on the heart rate, the temporal resolution was between 15 and 23 frames per cardiac cycle. The entire LV cavity and myocardium, including the epicardium, have been carefully included in the full volume data set ${ }^{(9)}$. The resultant $3 \mathrm{D}$-STE data sets were analyzed with EchoPAC software (GE Medical Systems).
- After setting two reference points at the LV base at the level of mitral valve and one at the apex on two orthogonal apical views at the frame of end-diastole, the software automatically tracked the endocardial and epicardial contours on subsequent frames through the entire cardiac cycle in three distinct vectors all the while. The endocardial border and myocardial thickness were adjusted manually to enhance boundary positioning and tracking ${ }^{(9)}$.

- The Area Strain values were automatically calculated by the software. The total maximum systolic AS value for the left ventricle was obtained, as well as the mean value for each segment (basal, middle, and apical). Other Strain measurements including circumferential, longitudinal and radial strain were also automatically calculated by the software. All data were collected and recorded.

- The management of the collected data was conducted during a period of one month and a half. The collected data were coded, processed and analyzed by using SPSS \{Statistical Package for Social Science\} computer software program version 20.

- The collected data were analyzed using frequencies, percentage, range, arithmetic means, and standard deviation.

- F-test was used for measuring statistical significance.

- Level of significance was adjusted at a level equal or less than 0.05 .

\section{Results}

Distribution of studied subjects according to age group in Table 1.

Table 1. Distribution of studied subjects according to age group

\begin{tabular}{|c|c|c|c|c|c|}
\hline Group & $\begin{array}{c}\text { Age by } \\
\text { years }\end{array}$ & No. & $\boldsymbol{\%}$ & Mean & \pm SD \\
\hline Group I & $\mathbf{2 0 - 2 9}$ & 60 & 33.3 & 21.9 & 2.2 \\
\hline Group II & $\mathbf{3 0 - 3 9}$ & 60 & 33.3 & 33.8 & 2.1 \\
\hline Group III & $\mathbf{4 0 - 5 0}$ & 60 & 33.3 & 44.7 & 2.8 \\
\hline Total & $\mathbf{2 0 - 5 0}$ & 180 & 100 & 33.4 & 9.7 \\
\hline \hline
\end{tabular}

Distribution of studied subjects according to sex in Table 2.

Table 2. Distribution of studied subjects according to sex

\begin{tabular}{|c|c|c|}
\hline & Male & Female \\
\hline Group I & 58 & 2 \\
\hline Group II & 57 & 3 \\
\hline Group III & 55 & 5 \\
\hline Total & 170 & 10 \\
\hline
\end{tabular}


Assessment of normal values of Area Strain by using Three-dimensional speckle-tracking Echocardiography Distribution of studied subjects according to Global Area Strain of age groups in Table 3.

Table 3. Distribution of studied subjects according to Global Area Strain of age groups

\begin{tabular}{|l|c|c|c|c|}
\hline & Group I & Group II & Group III & Total \\
\hline $\begin{array}{l}\text { Grea } \\
\text { Strain }\end{array}$ & & & & \\
\hline Number & & & & \\
\hline Mean & 60 & 60 & 60 & 180 \\
\hline \pm SD & -28.40 & -28.57 & -28.53 & 28.50 \\
\hline
\end{tabular}

Distribution of studied subjects of the three age groups according to basal segments of Area Strain in Table 4.

Table 4. Distribution of studied subjects of the three age groups according to basal segments of Area Strain

\begin{tabular}{|l|c|c|c|c|c|c|c|}
\hline \multirow{2}{*}{ Segments } & \multicolumn{2}{|c|}{$\begin{array}{c}\text { Group I } \\
(\mathrm{n}=60)\end{array}$} & \multicolumn{2}{c|}{$\begin{array}{c}\text { Group II } \\
(\mathrm{n}=60)\end{array}$} & \multicolumn{2}{c|}{$\begin{array}{c}\text { Group III } \\
(\mathrm{n}=60)\end{array}$} \\
\cline { 2 - 8 } & Mean & \pm MD & Mean & \pm SD & Mean & \multirow{2}{*}{ F-test } & \\
\hline Basal Anteroseptal & -26.40 & 7.79 & -25.93 & 10.66 & -27.83 & 7.95 & 0.71 \\
\hline Basal Inferoseptal & -25.90 & 8.41 & -26.38 & 9 & -23.88 & 6.76 & 1.65 \\
\hline Basal Inferior & -29.12 & 8.54 & -29.37 & 7.42 & -29.58 & 6.95 & 0.08 \\
\hline Basal Inferolateral & -18.85 & 22.30 & -19.68 & 19.21 & -24.03 & 15.64 & 1.27 \\
\hline Basal Anterolateral & -22.72 & 17.68 & -25.28 & 16.78 & -24.80 & 16.50 & 0.28 \\
\hline Basal Anterior & -33.50 & 8.55 & -29.12 & 12.38 & -30.38 & 10.56 & 3.01 \\
\hline Basal & -26.08 & 5.37 & -25.96 & 5.88 & -26.75 & 5.88 & 0.34 \\
\hline
\end{tabular}

Insignificant statistical difference between the three age groups

Distribution of studied subjects of the three age groups according to middle segments of Area Strain in Table 5.

Table 5. Distribution of studied subjects of the three age groups according to middle segments of Area Strain.

\begin{tabular}{|c|c|c|c|c|c|c|c|}
\hline \multirow{2}{*}{ Segments } & \multicolumn{2}{|c|}{$\begin{array}{c}\text { Group I } \\
(\mathrm{n}=60)\end{array}$} & \multicolumn{2}{c|}{$\begin{array}{c}\text { Group II } \\
(\mathrm{n}=60)\end{array}$} & \multicolumn{2}{c|}{$\begin{array}{c}\text { Group III } \\
(\mathrm{n}=60)\end{array}$} & \multirow{2}{*}{ F-test } \\
\cline { 2 - 8 } & Mean & $\pm \mathbf{S D}$ & Mean & $\mathbf{\pm S D}$ & Mean & & \pm SD \\
\hline Mid Anteroseptal & -32.02 & 7.63 & -29.22 & 8.53 & -32.18 & 8.33 & 2.27 \\
\hline Mid Inferoseptal & -30.07 & 5.91 & -29.88 & 5.60 & -29.83 & 5.47 & 0.01 \\
\hline Mid Inferior & -30.23 & 6.02 & -29.13 & 9.47 & -29.22 & 5.60 & 0.40 \\
\hline Mid Inferolateral & -31.02 & 7.12 & -30.55 & 10.13 & -31.47 & 7.95 & 0.13 \\
\hline Mid Anterolateral & -27.98 & 9.11 & -27.95 & 10.42 & -25.03 & 12.03 & 1.54 \\
\hline Mid Anterior & -27.52 & 11.24 & -25.08 & 12.54 & -25.17 & 12.32 & 0.75 \\
\hline Mid & -29.81 & 3.80 & -28.64 & 4.99 & -28.82 & 4.49 & 1.20 \\
\hline
\end{tabular}

Insignificant statistical difference between the three age groups

Distribution of studied subjects of the three age groups according to Apical segments of Area Strain in Table 6.

Table 6. Distribution of studied subjects of the three age groups according to apical segments of Area Strain.

\begin{tabular}{|c|c|c|c|c|c|c|c|}
\hline \multirow{2}{*}{ Segments } & \multicolumn{2}{|c|}{$\begin{array}{l}\text { Group I } \\
(\mathrm{n}=60)\end{array}$} & \multicolumn{2}{|c|}{$\begin{array}{l}\text { Group II } \\
(\mathrm{n}=60)\end{array}$} & \multicolumn{2}{|c|}{$\begin{array}{l}\text { Group III } \\
(n=60)\end{array}$} & \multirow{2}{*}{ F-test } \\
\hline & Mean & $\pm \mathrm{SD}$ & Mean & \pm SD & Mean & \pm SD & \\
\hline Apical Septal & -30 & 5.37 & -31.40 & 5.81 & -30.98 & 8.52 & 0.55 \\
\hline Apical Inferior & -34.40 & 6.63 & -33.73 & 7.12 & -34.73 & 6.24 & 0.35 \\
\hline Apical Lateral & -29.70 & 9.96 & -28.92 & 11.06 & -30.48 & 7.89 & 0.44 \\
\hline Apical Anterior & -21.73 & 9.29 & -21.65 & 10.97 & -23.55 & 10.03 & 0.68 \\
\hline Apical & -28.96 & 5.11 & -28.93 & 6.20 & -29.94 & 4.86 & 0.68 \\
\hline
\end{tabular}

Insignificant statistical difference between the three age groups

Mean values of Global Area Strain, Basal, Middle \& Apical Segments according to age groups in Table 7. 
Table 7. Mean values of Global Area Strain, Basal, Middle \& Apical Segments according to age groups

\begin{tabular}{|c|c|c|c|c|}
\hline Left Ventricular Values & Group I & Group II & Group III & F-test \\
\hline Global Area Strain & $-28.40 \pm 2.48$ & $-28.57 \pm 2.73$ & $-28.53 \pm 2.81$ & 0.07 \\
\hline Basal Segment & $-26.08 \pm 5.37$ & $-25.96 \pm 5.88$ & $-26.75 \pm 5.88$ & 0.34 \\
\hline Mid Segment & $-29.81 \pm 3.80$ & $-28.64 \pm 4.99$ & $-28.82 \pm 4.49$ & 1.20 \\
\hline Apical Segment & $-28.96 \pm 5.11$ & $-28.93 \pm 6.20$ & $-29.94 \pm 4.86$ & 0.68 \\
\hline
\end{tabular}

Insignificant statistical difference between the three age groups

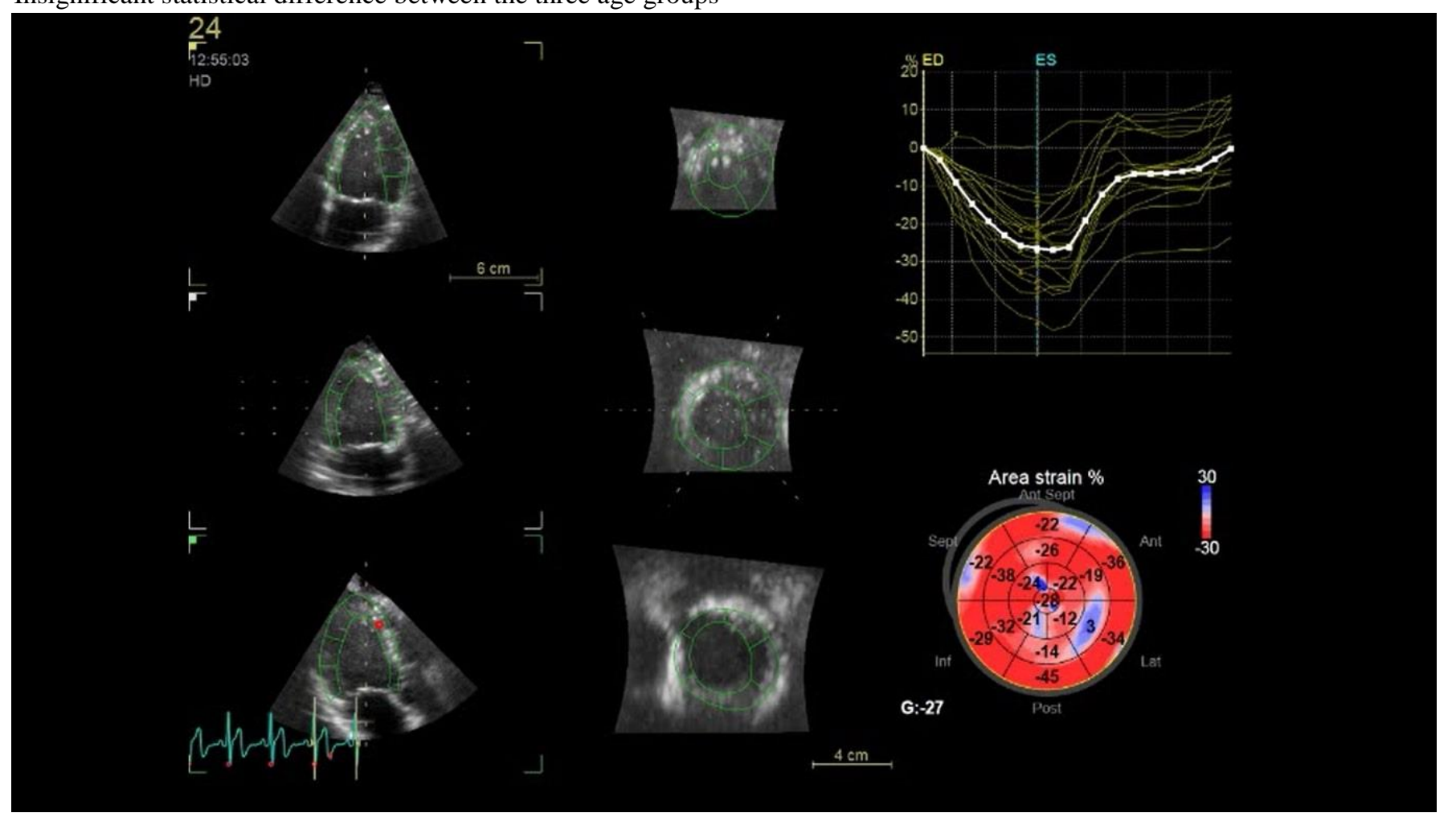

Figure 1. Global Area Strain of a 27 years old healthy male subject.

\section{Discussion}

Area Strain (AS) is an especially new method with a high clinical application potential. This new parameter consolidates an analysis of both the longitudinal and circumferential deformation of the left ventricle. This gives an estimate of the subendocardial surface deformation that is inversely proportional to the radial deformation (systolic thickening and diastolic thinning) of the wall of the ventricle, and is the first endocardium area to be affected by various cardiopathies ${ }^{(8)}$. The present study aimed to estimate the maximum normal systolic reference value of Area Strain in a sample of healthy subjects. The present study showed that the mean Global Area Strain at age group I, age group II and age group III were $-28.40 \pm 2.48 \%$, $-28.57 \pm 2.73 \%$ and $-28.53 \pm 2.81 \%$ respectively. The mean maximum systolic global area strain of the studied subjects was $-28.50 \pm 2.66 \%$. The difference between the mean values of global area strain of the three age groups was statistically insignificant.
These results are in agreement with Tadic et al. ${ }^{(10)}$, Kowalik et al. ${ }^{(11)}$, and Ternacle et al. ${ }^{(12)}$ who showed that the mean global area strain in healthy subjects was $-28.2 \pm 3.8 \%,-31.7 \pm$ $2.7 \%$ and $-31 \pm 4 \%$ respectively. The results of the present study are also in agreement with the results of Muraru $\boldsymbol{e t}$ al. (13) who used three-dimensional speckle-tracking echocardiography to measure left ventricular myocardial strain in healthy subjects and the reference value for 3D global area strain in his study was $-29 \%$ to $-35 \%$, with $-26 \%$ as a lower limit of normality.

The present study showed that the mean value for the basal segment of global area strain of 180 studied healthy subjects was $-26.26 \pm 5.69 \%$.

The present study also showed that the mean values for the middle and apical segments of global area strain of 180 studied healthy subjects were $-29.09 \pm 4.46 \%$ and $-29.27 \pm 5.41 \%$ respectively. The results of the present study for the basal, middle, and apical segments of the global 
area strain are not in agreement with those results by Pérez de Isla et al. ${ }^{(8)}$ who showed that the mean values in the basal, middle, and apical segments were $-38.42 \pm 7.58 \%$, $-38.74 \pm 6.34 \%$, and $-43.18 \pm 12.81 \%$, respectively. This disagreement is due to the difference in tracking algorithm and strain calculation among different vendors. Pérez de Isla et al. used a different ultrasound machine than the one used in the present study.

Badano et al. ${ }^{(14)}$ evaluated the inter-vendor consistency of 3D LV global strain values obtained using two distinct scanners. Badano et al. studied sixty patients (38 \pm 12 years, $64 \%$ males) with broad spectrumof ejection fractions (from 17 to $70 \%$ ). All of the patients underwent two acquisitions of 3D Left ventricular data set using VividE9 and Artida cardiac ultrasound machines. Global longitudinal, radial, circumferential and area strain values were acquired offline by using the corresponding softwares of 3D STE. Badano et al. concluded that the inter-vendor agreement of radial, circumferential and area strain measured with Artida and VividE9 was poor. Reference values ought to be specific for every system and baseline and follow-up data in longitudinal studies should be obtained through the same 3D STE platform.

Yuda et al. ${ }^{(15)}$ as well stated that values of three-dimensional LV strains by 3D speckle-tracking echocardiography are highly vendor-dependent.

Seo et al. ${ }^{(16)}$ showed that area strain was a useful tool for the quantification of segmental function.

The present study established the mean value for LV 3D Global Area strain parameter from a relatively big amount of healthy subjects. However, further studies are needed to define the normal values of Area strain in light of the poor intervene or agreement and reproducibility of strain measurements. Our present study showed excellent intraobserver reproducibility of the results and an overall acceptable interobserver reproducibility of strain measurements.

Our results showed positive correlations between global area strain values and each of the global circumferential and global longitudinal Strain. A negative correlation was presented between global area strain and global radial strain, and this is in agreement with the results of Kowalik et al. (11).

Area Strain seems a very promising new parameter which can be applied into routine clinical practice and this is in agreement with Galderisi et al. ${ }^{(17)}$ who used the parameter of global area strain by three-dimensional (3D) echocardiography to study 38 indigenous hypertensive patients.

Galderisi et al. ${ }^{(17)}$ showed that early changes in myocardial function could be recognized in these patients by global area strain. In addition, global area strain was a comprehensive parameter of myocardial systolic deformation and was highly sensitive to both changes of afterload and LV mass.

\section{Conclusion}

Global area strain obtained from 3D STE may be used clinically as an additional diagnostic method since Area Strain can provide a more global and comprehensive assessment of LV systolic function than traditional parameters. There is no statistical significance found between the mean values of global area strain, basal, middle and apical segments in the three age groups. The present study detected positive correlations between Global Area Strain values and each of the Global Circumferential and Global Longitudinal Strain. Negative correlations were detected between Global Area Strain and each of the Global Radial Strain and the Ejection Fraction.

\section{References}

1. Edvardsen T, Haugaa KH (2018): Strain Echocardiography: From Variability to Predictability. JACC: Cardiovascular Imaging, 11(1):35-37.

2. Langeland S, Rabben SI, Heimdal A, Gérard $O$ (2010): 4D Strain: validation of new 3D speckle tracking and left ventricular tool in simulated echocardiographic data. Eur J Echocardiogr., 11:2.

3. Maffessanti F, Nesser HJ, Weinert $L$ et al. (2009): Quantitative evaluation of regional left ventricular function using three-dimensional speckle tracking echocardiography in patients with and without heart disease. Am J Cardiol., 15:1755-62.

4. Nesser HJ, Mor-Avi V, Gorissen W et al. (2009): Quantification of left ventricular volumes using threedimensional echocardiographic speckle tracking: comparison with MRI. Eur Heart J., 30:1565-73.

5. Pérez de Isla $\mathrm{L}$, Balcones DV, Fernández-Golfín C et al. (2009): Three-dimensional-wall motion tracking: a new and faster tool for myocardial strain assessment: comparison with two-dimensional-wall motion tracking. J Am Soc Echocardiogr., 22:325-30.

6. Bouchez S, Heyde B, Barbosa D et al. (2016): In-vivo validation of a new clinical tool to quantify threedimensional myocardial strain using ultrasound. Int $\mathbf{J}$ Cardiovasc Imaging, 32(12):1707-1714.

7. Wen H, Liang Z, Zhao Y et al. (2011): Feasibility of detecting early left ventricular systolic dysfunction using global area strain: a novel index derived from threedimensional speckle-tracking echocardiography. European Journal of Echocardiography, 12(12):910916.

8. Pérez de Isla L, Millán M, Lennie V et al. (2011): Area Strain: Normal Values for a New Parameter in Healthy People. Rev Esp Cardiol., 64:1194-7.

9. Luo XX, Fang F, Lee AP et al. (2014): What can threedimensional speckle-tracking echocardiography contribute to evaluate global left ventricular systolic performance in patients with heart failure? Int J Cardiol., 172(1):132-7.

10. Tadic M, Cuspidi C, Backovic S et al. (2014): Highnormal blood pressure, functional capacity and left heart mechanics: is there any connection? Blood Press, 23(5):315-21.

11. Kowalik E, Kowalski M, Klisiewicz A et al. (2016): Global area strain is a sensitive marker of subendocardial damage in adults after optimal repair of aortic 
coarctation: three-dimensional speckle-tracking echocardiography data. Heart Vessels, 31(11):17901797.

12. Ternacle J, Bremont C, d'Humieres T et al. (2017): Left ventricular dyssynchrony and $2 \mathrm{D}$ and $3 \mathrm{D}$ global longitudinal strain for differentiating physiological and pathological left ventricular hypertrophy. Arch Cardiovasc Dis., 110(6-7):403-412.

13. Muraru D, Cucchini U, Mihaila S et al. (2014): Left ventricular myocardial strain by three-dimensional speckle-tracking echocardiography in healthy subjects: reference values and analysis of their physiologic and technical determinants. J Am Soc Echocardiogr., 27:85871.

14. Badano LP, Cucchini U, Muraru D et al. (2013): Use of three-dimensional speckle tracking to assess left ventricular myocardial mechanics: inter-vendor consistency and reproducibility of strain measurements. Eur Heart J Cardiovasc Imaging, 14(3):285-93.
15. Yuda S, Sato Y, Abe K et al. (2014): Inter-vendor variability of left ventricular volumes and strains determined by three-dimensional speckle tracking echocardiography. Echocardiography, 31(5):597-604.

16. Seo Y, Ishizu T, Enomoto Y et al. (2009): Validation of 3-dimensional speckle tracking imaging to quantify regional myocardial deformation. Circ Cardiovasc Imaging, 2(6):451-9.

17. Galderisi M, Esposito R, Schiano-Lomoriello V et al. (2012): Correlates of global area strain in native hypertensive patients: a three-dimensional speckletracking echocardiography study. European Heart Journal - Cardiovascular Imaging, 13(9):730-738. 